\title{
Studies on the Orientation of ACEL ZnS:Cu Particles in Applied AC Fields
}

\author{
T. G. Ireland and J. Silver \\ Centre for Phosphor and Display Materials, Wolfson Centre for Materials Processing Brunel University, Uxbridge, \\ Middlesex UB8 3PH, United Kingdom
}

\begin{abstract}
In the last sixty years the understanding of the mechanisms of alternating current electroluminescence (ACEL) in transition metaldoped zinc sulfides, in particular $\mathrm{ZnS}: \mathrm{Cu}$ particles has been the goal of all workers in the field. An in depth understanding of the crystallography and uncommon hemimorphic nature of these particles has lead to some understanding as to mechanism of light emission under an alternating current field. Hydrochloric acid etching of $\mathrm{ZnS}: \mathrm{Cu}$ particles has allowed an intimate study of the hemimorphic nature and high density of planar stacking faults present in the particles that are critical for ACEL to occur. This work using complimentary field emission scanning electron microscopy and digital optical microscopy shows that the alignment of the planar stacking faults of these particles relative to the applied electric field is critical for light emission. Perpendicular alignment of the stacking faults results in no emission of light; as the alignment gradually approaches parallel emission increases and at parallel reaches a maximum. Thus, for devices using these materials alignment of the particles with the electric field is most important to maximize light output. (C) The Author(s) 2013. Published by ECS. This is an open access article distributed under the terms of the Creative Commons Attribution 4.0 License (CC BY, http://creativecommons.org/licenses/by/4.0/), which permits unrestricted reuse of the work in any medium, provided the original work is properly cited. [DOI: 10.1149/2.008403jss] All rights reserved.
\end{abstract}

Manuscript submitted June 28, 2013; revised manuscript received December 17, 2013. Published December 27, 2013.

Over the last twenty years the extensive use of alternating current electroluminescent (ACEL) panels has seamlessly permeated into a wide range of modern technological devices, their introduction, exploitation and significance has gone unnoticed by unaware consumers, although their presence is visually evident. Such ACEL panels have seen use as backlights in both mobile phone keypads and their liquid crystal displays, computer and television screens, watches, control panels, automotive and aeronautical instrument panels. In addition they are widely used in advertising. Current ACEL devices are on the order of 50 to 1000 micrometers thick; this has allowed the previously mentioned appliances to be manufactured to their present slender dimensions. ACEL panels can be produced to have extreme flexibility, resistance to weathering, high brightness, low power consumption, long lifetimes, large area illumination, and are dimmable. These attributes has led to the use of ACEL panels for large area signage and commercial graphics, decorative architectural accent lighting, egress, and pathway lighting. ${ }^{1}$ The main advantage of using ACEL powder panels is the ability to screen print large areas (in various shapes) of thick layers of the relevant components that make up the device on a number of substrates. ${ }^{2}$

Destriau in 1936 was the first to observe ACEL. He studied a mixture of $\mathrm{ZnS}$ and $\mathrm{ZnO}$ powders suspended in castor oil between two electrodes emitted light when excited by an electric field. ${ }^{3}$ This was the first electroluminescent device that incidentally was an ACEL panel. The phenomenon of ACEL and an understanding of its mechanism with particular reference to $\mathrm{ZnS}: \mathrm{Cu}$ phosphor powders has still not been fully understood. The structural requirements of the $\mathrm{ZnS}$ host lattice that allows the manifestation of ACEL emission are complex and further complicated by the polytypism of its two polymorphs wurtzite and sphalerite. ${ }^{4,5} \mathrm{ZnS}$ is an extreme example of polytypism where the crystal has different stacking sequences of $\mathrm{Zn}-\mathrm{S}$ layers in two dimensions. ${ }^{6,7}$ For ACEL emission to occur in $\mathrm{ZnS}$; small amounts of $\mathrm{Cu}, \mathrm{Cl}$ will give green emission at lower frequencies and blue at higher frequencies whereas for $\mathrm{Cu}, \mathrm{Br}$ only blue emission is observed. These dopants and charge compensators are introduced during the synthetic processing, the conditions of which are of paramount importance to produce the exact polytypic modification.

Recent X-ray powder diffraction (XRPD) studies on commercial ACEL phosphor powders confirmed that as a result, of a two stage annealing during their synthesis, the phosphors were converted from the high temperature $\left(1024^{\circ} \mathrm{C}\right)$ hexagonal (wurtzite) structure to the low temperature cubic (sphalerite) polymorph having a high density of planar stacking faults. ${ }^{8}$ In contrast, electron and optical microscopy

${ }^{\text {z} E-m a i l: ~ j a c k . s i l v e r @ b r u n e l . a c . u k ~}$ studies on these phosphor particles after they were etched using hydrochloric acid (to remove their outermost surface) revealed their true crystal habit. ${ }^{8}$ The phosphor particles had the appearance of the hexagonal polymorph, which could be explained by the sphalerite pseudomorphing the earlier wurtzite ${ }^{9}$ such as reported by Fleet for a natural $\mathrm{ZnS}$ mineral from the Thomaston Dam, Connecticut, after undergoing a similar transformation from the hexagonal to cubic phase during synthesis. Furthermore, the high density of planar stacking faults along the cubic [111] axis were capped with pyramids on the (111) face this is consistent with unidirectional crystal growth (hemimorphic) originating at the face showing the pyramids. In addition many triangular etch pits are observed with their apices always pointing in the [111] direction toward the (111) face where the pyramids are viewed. ${ }^{8}$ Thus the (111) twinned phase of $\mathrm{ZnS}: \mathrm{Cu}$ - based phosphor is of importance in ACEL activity, ${ }_{10}^{10}$ whereas the etch pits may be necessary but not sufficient in order for ACEL to be produced. ${ }^{11}$ It should be noted that a cubic material has 4 [111] axis. In the case of these crystals the [111] axis we are referring to lies along original $\mathrm{c}$ axis directions of the hexagonal phase (before it was converted to the cubic phase). In the hexagonal crystals this was the axis the main growth took place along. ${ }^{8}$ Throughout this paper when we refer to the [111] axis as explained above we do not refer to the other 3 [111] cubic axis.

There have been many papers published with regard to the mechanism and origin of ACEL in $\mathrm{ZnS}: \mathrm{Cu}$ particles, these have provoked much debate and speculation. One of the most widely held interpretations is that put forward by Fischer who conducted microscope studies on $\mathrm{ZnS}: \mathrm{Cu}$ particles in experimental cells during operation. ${ }^{12,13} \mathrm{He}$ suggested that the presence of linear $\mathrm{Cu}_{2} \mathrm{~S}$ precipitates formed at the sites of the extensive twinning planes created by the two crystallographic phases during crystal growth and this may be responsible for the light emission during ACEL. Fischer proposed that there would be deformation of the applied electric field that would be concentrated and enhanced at the $p$-type semiconductor linear $\mathrm{Cu}_{2} \mathrm{~S}$ precipitates within the insulating $\mathrm{ZnS}$ particle. This would induce injection of electrons and holes from opposite ends of the linear $\mathrm{Cu}_{2} \mathrm{~S}$ precipitates into the $\mathrm{ZnS}$, in the alternating field electrons and holes are injected alternatively at the same end. Each cycle would consist of holes trapped at luminescent centers, the electrons would remain mobile or captured at shallow electron traps, followed by the emission of light as the electrons recombine with the holes. This model found acceptance due to the observation by Fischer ${ }^{12,13}$ of localized double comets of light that were emitted alternately on field reversal along the direction of the stacking faults and subsequently reported by other workers. ${ }^{14,15}$ In addition to localized comets of light, spots and patches have also been observed indicating that the light emission is inhomogeneous in this material. ${ }^{14-16}$ 
Although the Fischer model is still widely accepted, a number of studies have been conducted in an attempt to confirm the existence of the postulated $\mathrm{Cu}_{2} \mathrm{~S}$ phase and in turn the exact mechanism of the ACEL phenomenon but these have not produced a definitive answer. For example Ono et al., ${ }^{17}$ reported the detection of copper sulfide needles in the form of $\mathrm{Cu}_{2} \mathrm{~S}$ by means of $\mathrm{X}$-ray absorption near edge structure. Guo et al., ${ }^{18}$ observed a higher concentration of copper in the twin boundary areas and crystal streaks of sphalerite using high resolution electron microscopy and scanning electron microanalysis. A recent article by Bridges and co-workers, ${ }^{19}$ maintained that copper sulfide is present in the form of tiny CuS-like clusters by means of extended X-ray absorption fine structure. They also found during milling that the $\mathrm{CuS}$ precipitates were removed from the phosphor matrix by cleavage along the stacking planes,$^{20}$ they also found that degradation and subsequent rejuvenation of ACEL emission was possible. ${ }^{21}$ They found that thermal treatment above $240^{\circ} \mathrm{C}$ produced a significant degradation and change in spectral shape whereas below this temperature there was not a change, this indicated that two mechanisms were operative. Fern et al., found thermally quenched ACEL $\mathrm{ZnS}: \mathrm{Cu}$ powders showed the $\mathrm{Zn}$ k-edge to be of reduced intensity. Hence the electronic properties were modified. In ACEL panel measurements found using synchrotron powder X-ray diffraction measurements that there was a large variation in crystallography of individual phosphor grains. ${ }^{22}$ However, though others considered linear defects ${ }^{13,14}$ and Fischer also considers copper sulfide defects ${ }^{13}$ they found no direct evidence for copper sulfide needles. ${ }^{13.14}$ Thus the precise nature of the role of copper sulfide clusters and the charge generation process presently remain open to conjecture. It is worth considering that typical $\mathrm{Cu}$ concentrations in ACEL phosphors are typically low $\sim 0.1 \%$ (due to the low solubility of $\mathrm{Cu}$ in $\mathrm{ZnS}$ ). Thus, any copper sulfide defects must be small, certainly too small to be observed in the $1960 \mathrm{~s}$ and probably too small even to be imaged even today. The very latest transmission microscopes have a resolution of around $0.2 \mathrm{~nm}$ and it would be a good idea to check for such structures on well prepared samples.

Oranovskij and Khmelinin found that ACEL only occurred if the direction of the field was perpendicular to the pseudo $c$-axis of the $\mathrm{ZnS}: \mathrm{Cu}$ crystal, in other words along the planar stacking faults. ${ }^{23}$ This view that the orientation of $\mathrm{ZnS}: \mathrm{Cu}$ particles relative to the applied field direction is of significance with regards to ACEL emission has also been supported by other workers. ${ }^{8,13-17}$

Low magnification optical microscope studies reveal that commercial ACEL panels when excited with an electric field have a speckled appearance, the particles have a diameter between 10 to 50 micrometers are observed to emit with different intensities independent of particle size. ${ }^{24}$ A number of particles in all ACEL panels do not display electroluminescence. When the phosphor layer in ACEL panels is excited with $366 \mathrm{~nm}$ light all the particles are observed to emit light with equal intensity.

The papers noted above that refer to the importance of alignment of $\mathrm{ZnS}: \mathrm{Cu}$ particles in an applied AC field have all suffered from a large degree of uncertainty toward the exact orientation of the particles. We have previously demonstrated that the hydrochloric acid etching studies on ACEL ZnS:Cu phosphor powders reveals the true crystal habit of the particles. ${ }^{11}$ Building on this work it was possible to observe their exact alignment with respect to the applied AC electric field if the correct ACEL cell architecture was used and we report the results of these studies herein. In this work we used hydrochloric acid etched particles in conjunction with an inter-digitated linear track electrode ACEL panel. The device did not require transparent conducting electrodes as the architecture had the advantage of allowing the uncovered etched particles to be observed directly under an applied AC electric field. The open aspect of the device allowed the exact position of all the ACEL emitting particles relative to the applied AC field to be ascertained with a high degree of accuracy. By utilizing complimentary optical and electron microscopy techniques the combination of hydrochloric acid etched particles and the panel architecture also offered opportunity to gain insight into the surface structures to see if they have any influence on the observed emission of light.
Also some of the ACEL $\mathrm{ZnS}: \mathrm{Cu}$ particles were etched with sulphuric acid to reveal different surface features to the hydrochloric etched samples, these will be briefly discussed herein.

In addition to these experiments etched ACEL $\mathrm{ZnSCu}$ phosphor particles were set in a thin layer of adhesive whereby the particles extended above the adhesive surface. These particles were then mapped by FESEM imaging to ascertain their orientation and milled to reveal their interiors.

\section{Experimental}

Materials. - The following materials were used in this work without further purification. Uncoated type $728 \mathrm{ZnS}$ :Cu ACEL powders (Osram Sylvania, Towanda, USA), hydrochloric acid (Merck, Poole, UK, AnalaR, 0.38 M), sulfuric acid (Aldrich, Gillingham, UK, 9598\%), adhesive and hardener (Loctite, Henkel Ltd, Hemel Hempstead, UK).

Particle size and $\mathrm{Cu}$ concentration. - The particle sizes of the type 728 ACEL phosphor used in this work were in the range 30 to $35 \mu \mathrm{m}$ which is in line to the $31.4 \mu \mathrm{m}$ found by others, ${ }^{24}$ who also reported a copper concentration of 0.077 (at \%) from atomic absorption measurements.

Acid etching. - The hydrochloric etched ACEL ZnS:Cu phosphor particles were prepared by placing the uncoated phosphor $(1.0 \mathrm{~g})$ into a plastic centrifuge tube $(50 \mathrm{~mL})$, hydrochloric acid $(0.38 \mathrm{M}, 10 \mathrm{~mL})$ was then added to the tube which was tightly sealed with the cap. The tube was gently shaken, and then the cap was slowly released to allow the escape of the evolved gas, the tube was resealed and the process was continually repeated over a period of 10 minutes. The particles were then allowed to settle. The hydrochloric acid was decanted from the particles then deionised water $(50 \mathrm{~mL})$ was added to the tube which was resealed and shaken, the particles were again allowed to settle and the water was decanted off, this was repeated a further 3 times. After washing the particles were filtered and dried in an oven at a temperature of $140^{\circ} \mathrm{C}$ for a period of 20 minutes. During the hydrochloric acid etching no measures were taken to minimize surface oxidation other than sealing the tubes (however as the volume of oxygen in the tube was around $8 \mathrm{~mL}(20 \%$ of $40 \mathrm{~mL})$ and that in the acid is also small so we would expect less than $0.014 \mathrm{gm}$ of $\mathrm{O}_{2}$ to react first with the copper dissolved in the hydrochloric acid from the particles rather than cause an oxide coating on the particles. Once the particles were oven dried they were stored under nitrogen; when not being studied the particles were always stored under nitrogen between use. So any oxide formed on the surface would not propagate on standing. With most uncoated ACEL phosphor particles surface reactions occur in a short time period usually in the form of carbonate deposits from $\mathrm{CO}_{2}$ in air. ${ }^{25}$ There is no evidence that an oxide layer would disguise the shape of perceived light due to mere complex changes in refractive indexes.

A sulfuric acid etched ACEL $\mathrm{ZnS}: \mathrm{Cu}$ sample was also prepared in the same manner as the hydrochloric acid etched samples, (except an etching time of 40 minutes was used).

Inter-digitated electrode substrate.- A flexible Mylar coated paper substrate was lithographically printed with miniaturized interdigitated silver electrode circuits, the linear tracks and gaps having widths of 100 and 200 micrometers respectively. The resulting device did not require transparent conducting electrodes. This would allow FESEM studies of the etched ACEL $\mathrm{ZnS}: \mathrm{Cu}$ particles after optical imaging and identification of individual particles before and after application of alternating electric field and subsequent light emission from said particles. A number of tracks on the device were rendered non-conductive. A thin film of adhesive was applied to the surface of the substrate with a heavy copper roller; the layer was thin enough for the majority of particles to extend above the adhesive surface after fixing the particles to the circuitry for repeated transportation between the optical microscope, FESEM and storage. The phosphor particles 
were scattered onto the adhesive film; then after applying an alternating electric field, the adhesive was allowed to cure. It should be noted that the setup to access the particles has some drawbacks as the gaps between the tracks are $200 \mu \mathrm{m}$ and the typical ACEL particles are 30 to $35 \mu \mathrm{m}$ in size. An old style ACEL panel available on the market would only be around 150 to $200 \mu \mathrm{m}$ thick and would contain one or two dielectric layers. Such devices usually run at $110 \mathrm{~V}$ and $400 \mathrm{~Hz}$. We acknowledge that the gap we are using is $200 \mu \mathrm{m}$ and our particles do not bridge the gap (in common with market devices) and we have no dielectric layer present. However the intent in this work using the inter-digitated electrodes was to carry out fundamental studies. Obviously optimized commercial structures would have $\mathrm{BaTiO}_{3}$ in the outer layers. In this work we used much higher voltages $(300 \mathrm{~V}$ RMS).

Digital optical microscopy. - A Zeiss Axioskop MAT optical microscope with a Zeiss AxioCam MRc5 digital camera was used to image individual ACEL phosphor particles. The particles were optically imaged using (a) incident and (b) transmitted white light, (c) ACEL emission with white light transmittance and finally (d) pure ACEL emission. Magnifications of x25, x100 and x200 were used to obtain the images. The particles that had orientated in the applied electric field were then identified from those that had not been subjected to the electric field in the gaps between the non-conductive tracks. It should be noted that index matching was not used in the optical microscopy studies for two main reasons; firstly the optical images before alignment in the field were in a wet adhesive making index matching impractical, after alignment the adhesive was allowed to cure. Secondly the substrate was imaged using both microscopy techniques many times over a period of six months, removing the index matching compound every time FESEM imaging was used would seriously affect the adhesive and the integrity of the phosphor positioning.

Field emission electron microscopy. - For the FESEM studies a Zeiss Supra VP35 instrument was used for direct structural comparison with the optical images of the identified individual particles on the device. The device with the attached hydrochloric acid etched phosphor particles was imaged without a conductive coating. The images were collected with either a secondary electron detector using an accelerating voltage of $10 \mathrm{kV}$ to study surface detail or a secondary backscattered detector and $30 \mathrm{kV}$ accelerating voltage. The latter conditions were applied to allow the electrons to penetrate deeper into the surface of the surrounding adhesive and visualize a larger area of the particle profile of the submerged phosphor particles.

\section{Results and Discussion}

Generally ACEL ZnS:Cu particles observed at low magnification with optical or electron microscopes appear to have a morphology composed of flat surfaces with rounded edges and corners. It is possible to see striations along some particle surfaces, these are due to planar stacking faults perpendicular to the cubic [111] axis indicating crystal growth along the [111] axis this allows an estimation to be made as to the orientation of the particles.

When comparing the morphology of synthetically grown ACEL $\mathrm{ZnS}: \mathrm{Cu}$ particles and naturally formed sphalerite and wurtzite, and their mixed phases there do not appear to be any apparent similarities. For example Figure 1a presents a FESEM image of synthetic ACEL $\mathrm{ZnS}: \mathrm{Cu}$ particles that have a smooth rounded surface morphology with many flat faces. Figure $1 b$ presents an SEM study of a natural sphalerite tetrahedron epitaxic on hexagonal wurtzite. ${ }^{26}$ It is obvious from Figures 1a and $1 \mathrm{~b}$ that there is not any similarity between the synthetic and natural $\mathrm{ZnS}$ samples.

Natural wurtzite displays a hemimorphic growth habit, by analogy the mineral resembles a cauliflower or pyramid, it has distinct top and basal structural features. Hemimorphism in wurtzite is caused by the crystals $\mathrm{SZn}_{4}$ tetrahedra that are stacked in a layered structure and orientated in one direction producing hexagonal (six fold rotational) symmetry. The symmetry is broken in the perpendicular direction to

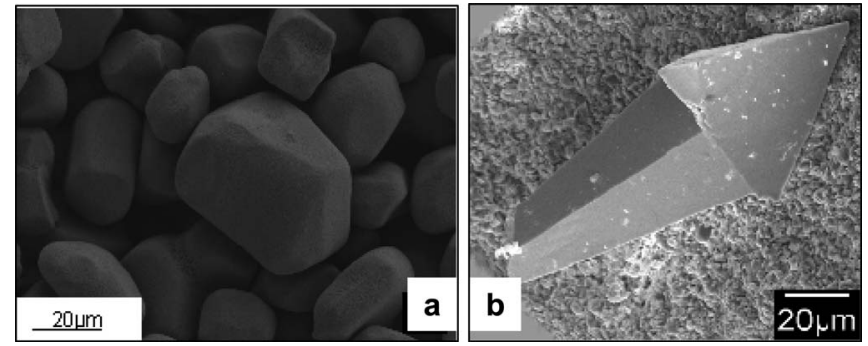

Figure 1. (a) FESEM image of synthetic ACEL $\mathrm{Zn}: \mathrm{Cu}$ particles (b) SEM study of a natural sphalerite tetrahedron (triangle top right) epitaxic on hexagonal wurtzite. ${ }^{26}$

the major axis, there is no symmetry element such as a mirror or twofold rotational axis perpendicular to the major axis and crystal faces at the top of the crystal are not repeated at the bottom; this produces a hemimorphic crystal. As mentioned previously ${ }^{8}$ hydrochloric acid etched ACEL ZnS:Cu particles revealed that a hemimorphic structure is present below the surface topology, an example is shown in the FESEM study shown in Figure 2. Hydrochloric acid etching of the ACEL $\mathrm{ZnS}: \mathrm{Cu}$ particles allowed a facile evaluation as to the exact orientation of all individual particles relative to an applied electric field and the nature of the emitted light using low magnification optical microscopy imaging. Due to this ability to discriminate between individual particles it may be possible to use complimentary FESEM examination to intimately identify any structural features that were related to the light emission during the optical studies.

Two low magnification aligned optical images of the ACEL device showing an area of the bare inter-digitated substrate, (Figure 3a) and an area where the phosphor particles have been laid down and are exhibiting electroluminescence are presented in Figure $3 \mathrm{~b}$. In Figure 3 are the fixed hydrochloric acid etched ACEL $\mathrm{ZnS}: \mathrm{Cu}$ phosphor particles that can be seen as the two horizontal linear blue light emitting regions in Figure $3 b$, during excitation (from an applied alternating electric field of $320 \mathrm{~V}$ RMS and a frequency of $10,000 \mathrm{~Hz}$ ). A fully functioning conductive track is clearly seen as the horizontal dark line running between the blue light emitting tracks of the etched phosphor particles approximately one third the way down in the figure. The rest of the image shows areas where the conductive tracks were disabled, hence the particles do not emit light. A dark vertical register mark

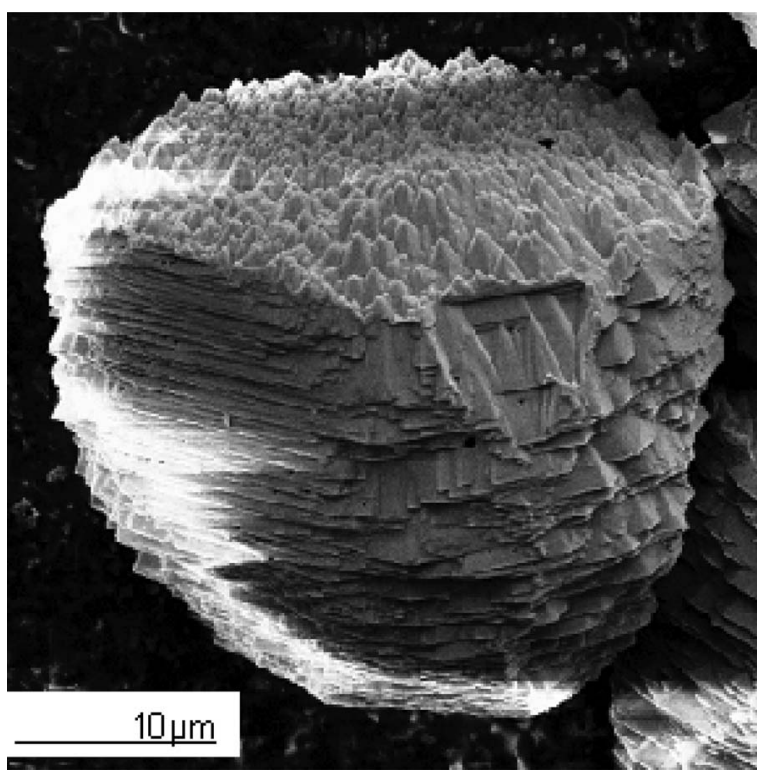

Figure 2. An FESEM image of a single Osram Sylvania ACEL ZnS:Cu phosphor particle that was hydrochloric acid etched displaying the hidden hemimorphic crystal habit of synthetic $\mathrm{ZnS}$. 


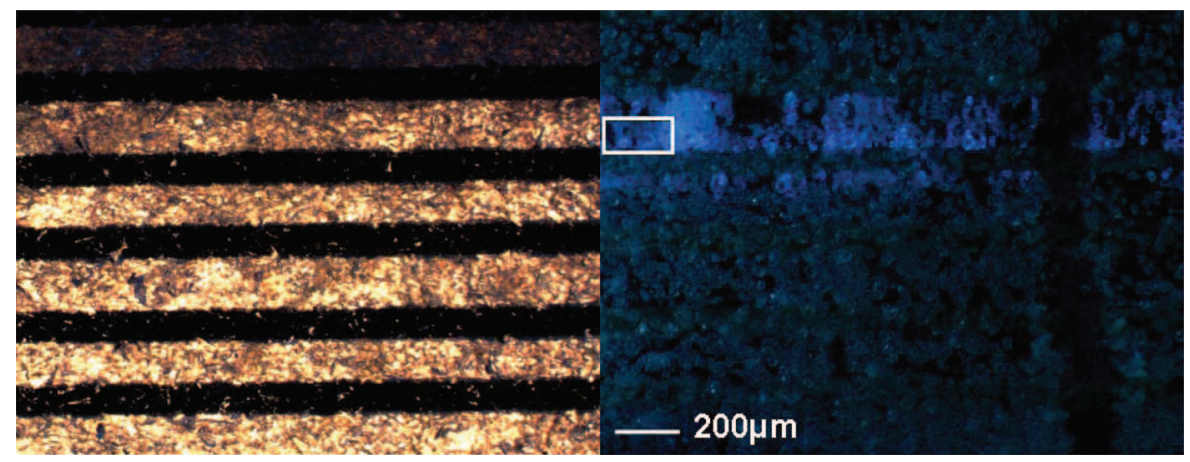

Figure 3. Optical microscope images (a) left image from an area of bare interdigated substrate showing where the tracks are under transmitted light; (b) right image the tracks are now covered with ACEL ZnS:Cu phosphor particles (that were etched with hydrochloric acid) under applied electric field exhibiting electroluminescence.

is seen to the right of center of the image (in Figure $3 b$ ) where the surface particles were removed by gently scratching the surface. This mark was used for recognition and navigation during microscopy examinations and has no other relevance in this study. The direction of applied AC electric field in all images is in the plane of the paper and in the vertical direction (i.e. alternatively up and down in relation to the top and bottom of the page).

In Figures $4 \mathrm{a}$ and $4 \mathrm{~b}$ higher magnification optical microscope images of the region of interest in Figure 3 are displayed (using incident and transmitted white light respectively). The interface between the conducting track and gap is represented by the demarcation of dark and light areas across the lower region of the images respectively. Surface features such as planar stacking faults, surface indentations, and pyramids are seen on some phosphor particles. Due to the depth of field at this magnification and the large particle size distribution ( 5 to $40 \mu \mathrm{m}$ ) not all particles are in sharp focus. The hemimorphic nature of some particles is clearly evidenced by their flat tops that are capped with silhouetted pyramids and terminated with pointed basal pedions when viewed side on.

The optical study in Figure $4 \mathrm{c}$ is of the same ACEL phosphor particles under an applied alternating electric field (320 V RMS, $10,000 \mathrm{~Hz}$ ) and transmitted white light, the majority of particles are shown to be emitting blue light concentrated at opposite sides of the crystals. It should be noted that at low frequencies the particles emit green light that gradually shifts to blue wavelengths at higher frequencies which is the case in this study. Also lines (or streaks) of emitted light in our case parallel to the fault planes are observed in some crystals in Figures 4, 5 and 6 (particularly the latter which shows two crystals under higher magnification), possibly explaining the "streaks" of light observed by others in previous studies. ${ }^{12-16,24,27}$ A FESEM micrograph is presented in Figure $4 \mathrm{~d}$ of the phosphor particles shown in the previous optical images in Figure $4 \mathrm{a}$ to $4 \mathrm{c}$, the exact orientation of all particles is now clearly revealed. The brightest ACEL emitting particles (see Figure 4c) are shown to be oriented with cubic [111] axis at various angles, generally in a perpendicular direction to the applied alternating electric field that radiates across the gap between conducting tracks. The planar stacks in these orientations are parallel to the applied field. An example of an aligned and non-aligned particle is shown by the vertical arrow (aligned) and horizontal arrow (non-aligned) depicted in the images presented in Figure 4.

If the parallel alignment of the planar stacking faults with the applied electric field approaches coincidence, the emission of light will be observed to reach a maximum intensity. As the alignment of the particles gradually becomes perpendicular to the applied electric field the brightness decreases. When the electric field is experienced by a particle that is fully aligned to it the field induces a field within the particle that is opposite to it. If the particle is partially aligned only a partial alignment with field will occur, other particles that touch such partially aligned particles may themselves align to the secondary fields and thus generate a small amount of light. Therefore a particle has to be isolated and non-aligned with the applied electric to be rendered totally ACEL inactive. This is an important point and explains findings by other workers who observed that a number of particles are non-emitting. ${ }^{24}$ The use of hydrochloric acid etched phosphor particles would have been of great assistance to the previous study.

In Figure $4 \mathrm{c}$ the some of the ACEL emitting particles take a different orientation where their [111] axis is still perpendicular to the field but instead of it lying in the plane of the paper it is also perpendicular to the paper. In this orientation the (111) planes also lie parallel to the field and these particles when seen end on show light as a torus or a homogeneous luminescence and do not show linear streaks of light when viewed from above. They would show linear streaks if seen from the side i.e. along the direction of the paper. This is can be explained on examination of the FESEM study presented in Figure 4d where the pyramid capped (111) face of some phosphor particles are observed to be oriented upwards toward the microscope aperture for example the particle at the bottom right of Figure 5c. This orientation still allows alignment of the [111] stacking planes with the applied electric field whilst they are obscured from view, therefore, the emissions are observed to have a different form. From these microscope images it is easy to ascertain that the particles exhibiting very little or no light emission have their planar stacking faults aligned perpendicular to the electric field direction by varying degrees. This is demonstrated by some of the particles shown in Figure 4a to $4 \mathrm{~d}$ where the [111] plane is almost perpendicular to the electric field direction.

In Figure 5a to 5d detailed area studies of those shown in Figure $4 \mathrm{a}$ to $4 \mathrm{~d}$ (just to the middle right of center) are presented. The images are dominated by two large particles both laying on their sides, displaying planar stacking faults perpendicular to their [111] growth direction both are excellent examples of the anisotropic habit of $\mathrm{ZnS}$ crystals. The two largest particles in the images are shown to have significant alignment that is parallel to the applied electric field. Both particles display in Figure 5c areas of homogeneous ACEL light emission with points and linear streaks of stronger light running parallel to the striations all around the edges. The FESEM image shown in Figure 5d allows the identification of some surface features where the light is observed to originate.

Figures $6 \mathrm{a}$ and $6 \mathrm{c}$ show optical microscope magnifications of the large triangular shaped particle to the right of Figure $5 \mathrm{c}$ and, Figure $6 \mathrm{~b}$ and $6 \mathrm{~d}$ are their complimentary FESEM images. The optical images show that the prominent linear streaks of light are formed of individual points of light, of which one point appears to be the brightest with a successive tailing off of light intensity along the streak, this detail probably appeared as the comets described in other studies. ${ }^{12-16,24,27}$ The linear streaks of light are shown against a background of dimmer light that is composed of numerous points of light forming small groups with varying intensities. When comparing the FESEM images it is clear that these linear streaks of light appear to originate from elongated etched incisions along the planar stacking faults. The strong points of light at the head of these streaks may emanate from etch pits or sharp rounded features observed in the high magnification FESEM images. These stacking faults appear to act as waveguides, steering the light away from its area of origin, again giving the impression that they are comets.

It is both difficult but also dangerous (from the point of view of over interpretation) to estimate the size of the light streaks and points. This is also true because of the light originating in the stacking faults. 

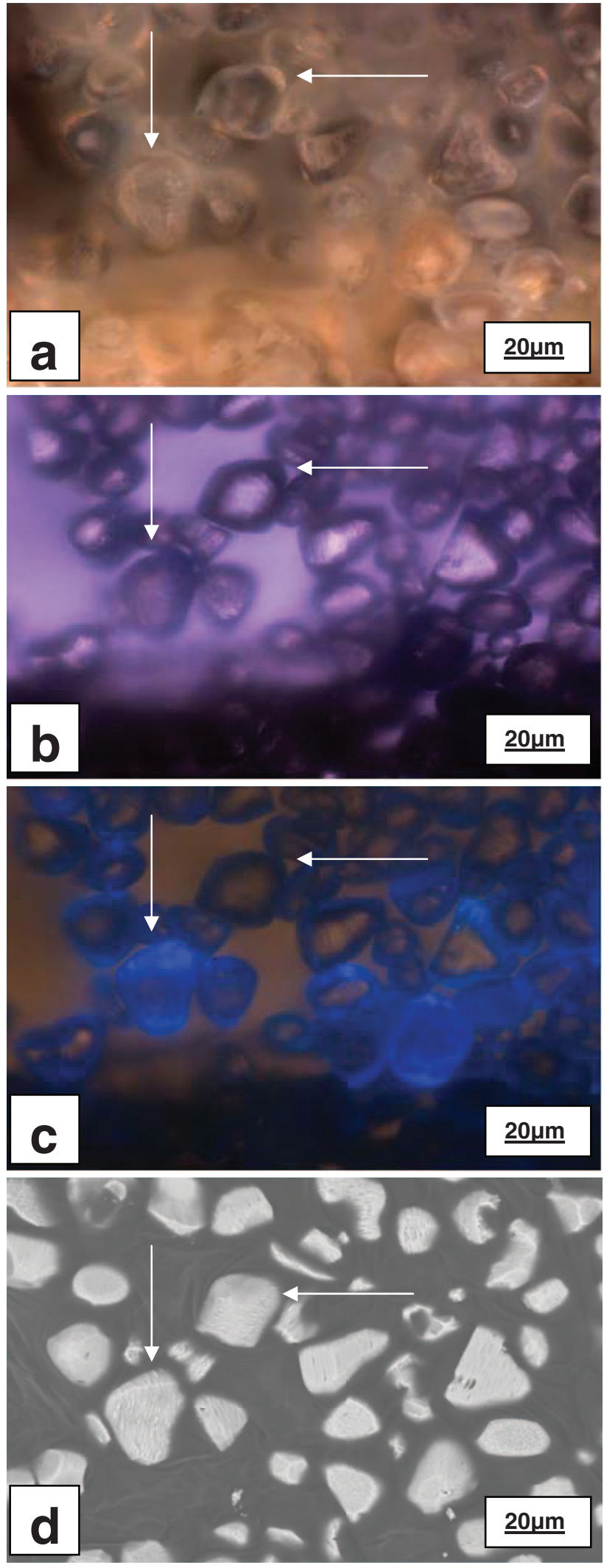

Figure 4. Higher magnification studies of microscopy images from region of interest shown in Figure 3 (a) transmitted white light, (b) reflected white light, (c) ACEL emitted light plus transmitted white light, (d) secondary backscattered FESEM image of (a) to (c). Magnification x200, scale bar $20 \mu \mathrm{m}$. The two arrows shown in Figure 4 (a-d) indicate the direction of alignment of the layers in the designated particles.
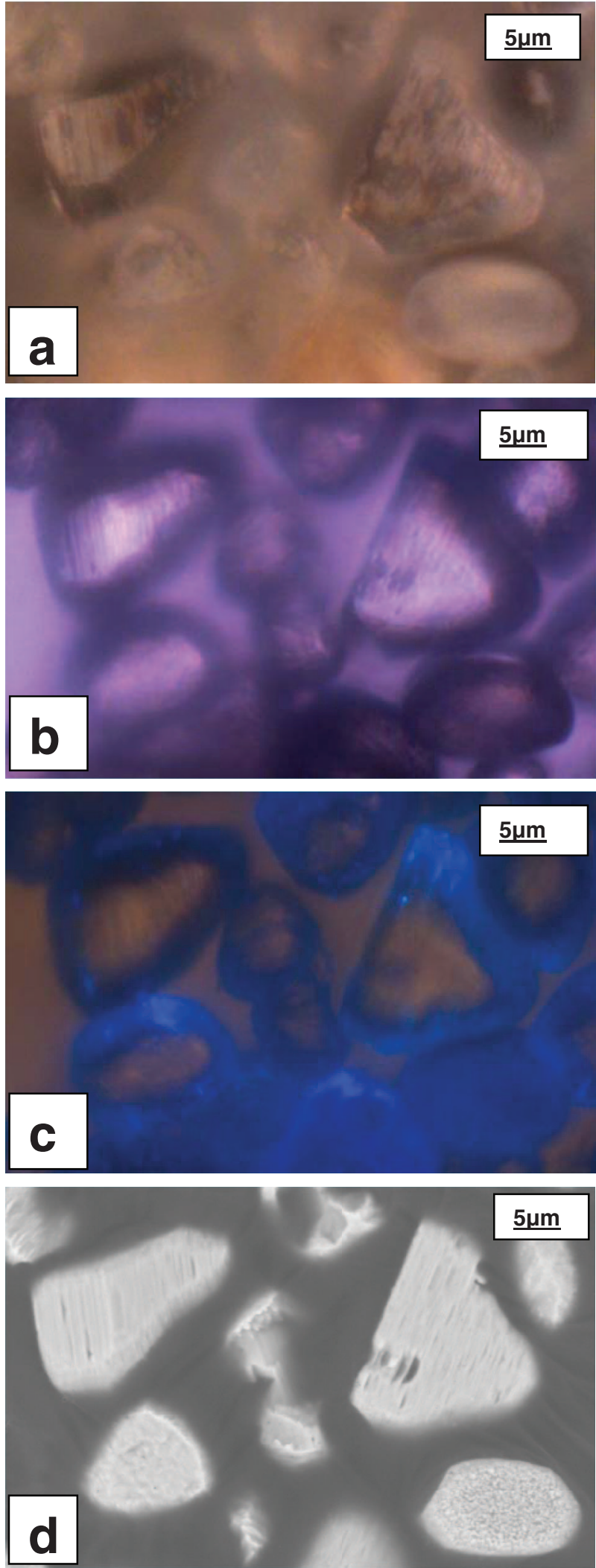

Figure 5. Detail from the right of center of the images shown in Figure 4.

The dimensions of the observed light emissions may appear larger than they actually are as the light is also partially diffuse, the diameter of the points and the width of the linear light streaks are in the region of 100 to $200 \mathrm{~nm}$ respectively, but at this time we do not think it 

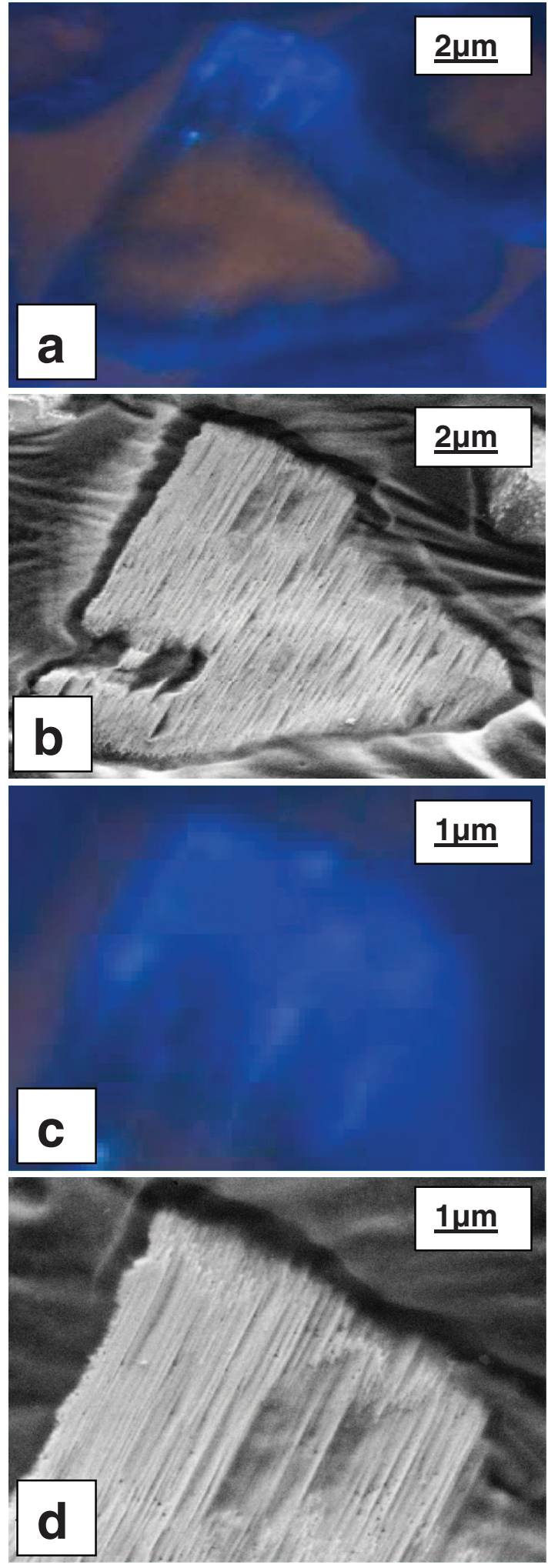

Figure 6. (a) Optical image of a hydrochloric acid etched ACEL $\mathrm{ZnS}: \mathrm{Cu}$ phosphor particle emitting light under an applied electric field (b) an FESEM study of (a); (c) and (d) are higher magnification details of the top corner of (a) and (b) respectively.

valuable to speculate on this. It is also worth noting that emission from electron-hole recombination should occur in very small regions (such as point defects) so that larger points or streaks must arise from the way the light exits the particle.
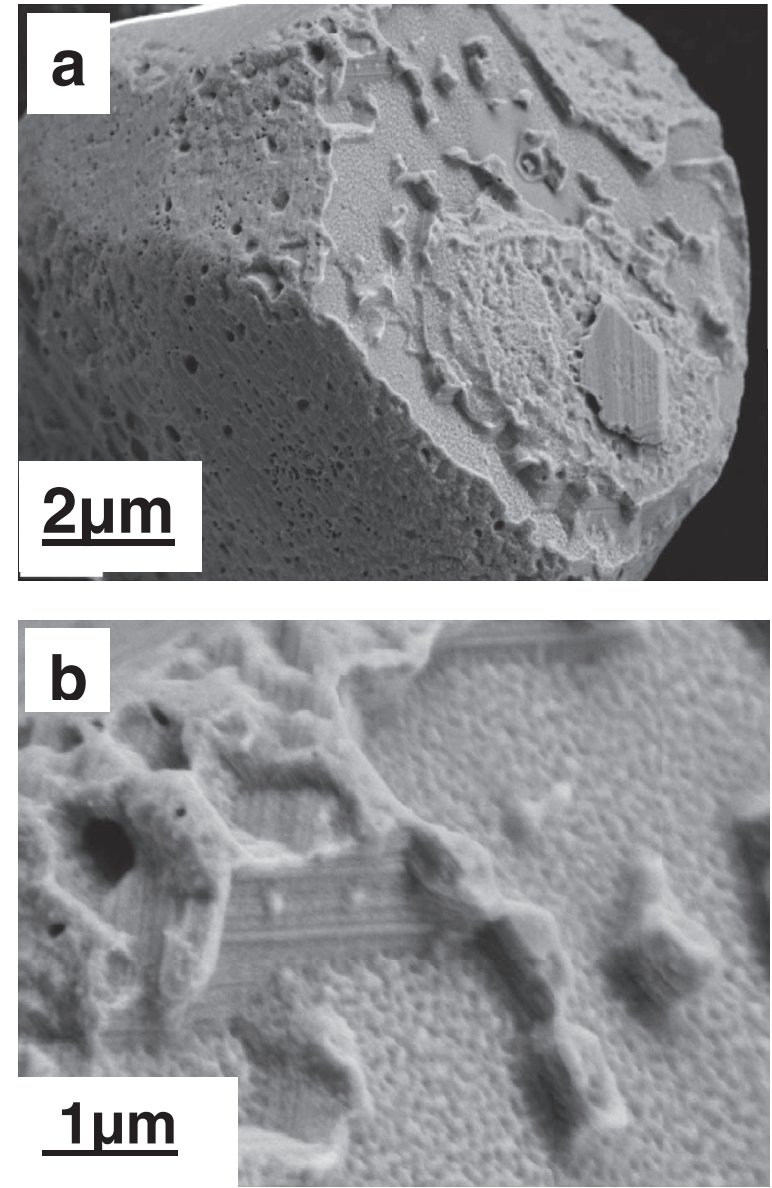

Figure 7. FESEM images of (a) sulfuric acid etched ACEL ZnS:Cu phosphor particle showing newly revealed structures different from those seen using a hydrochloric acid, (b) higher magnification study of top center from (a).

In Figures 7a and 7b FESEM images of an ACEL ZnS:Cu particle that has been treated with a different etchant, in this case sulfuric acid are presented. It is viewed looking down onto the (111) face and it is clear that this etchant has exposed different features within the particle to those revealed by hydrochloric acid etching. Of particular interest is the absence of pyramids that were found to be present on this face of the phosphor particles after hydrochloric acid etching. The flat expanse of rounded structures is also a new type of feature. Of particular relevance to the reported ACEL light emissions being in the form of spots or linear streaks of spots, ${ }^{17-19,24,27}$ it is apparent from the FESEM images in Figures $7 \mathrm{a}$ and $7 \mathrm{~b}$ that where an exposed face of the (111) plane is first attacked by the sulphuric acid it appears to first form faults across its surface (almost like the layers seen on the hydrochloric acid etch particles) but not the same as they are now in the (111) plane face. These are superimposed with evenly spaced circular protrusions (or spots) of material running along the length of the fault. Each spot of material is approximately $100 \mathrm{~nm}$ in diameter, a size similar in dimension to the spots of light observed in the optical images in this work seen in Figures 6a and 6c. These spots appear to act as a nucleus for the larger lumps shown in Figure 7 near these features. It is difficult to speculate as to the origins of the spots, does this mean each (111) face really contains smaller defined structures or are the spots just a result of the way that sulfuric acid attacks the (111) face. It is the (111) face that manifests pyramids at the extremity of the particle. Sulfuric acid attack on the pyramids may well etch these back to smaller structures which manifest as these "spots".

Moreover, it should be noted that the light appears to originate near to the surface in the optical microscope images reported in this study (see Figures 6a and 6c), this may be due to the focusing of 

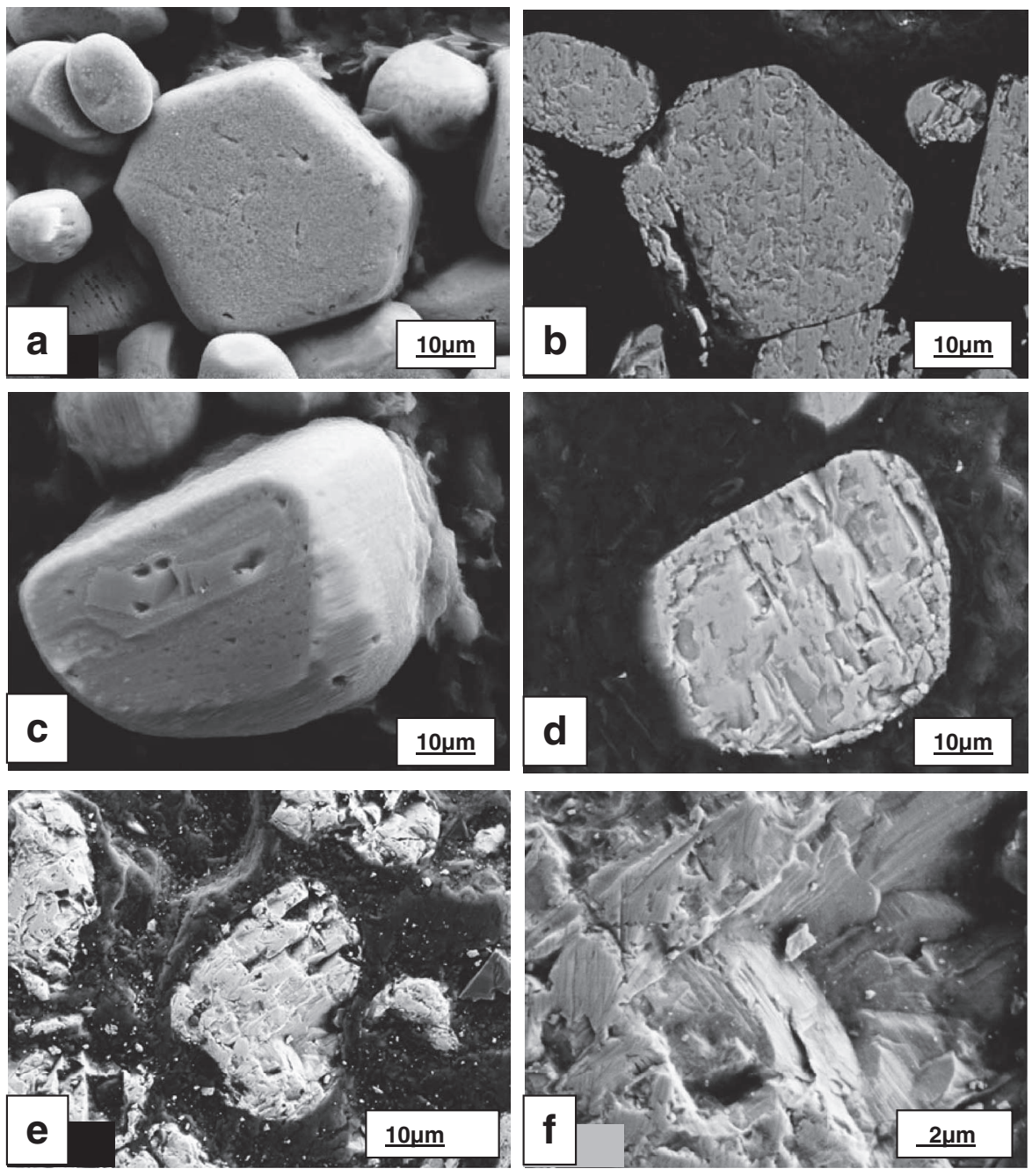

Figure 8. FESEM images of hydrochloric acid etched pre-milled and milled ACEL $\mathrm{ZnS}: \mathrm{Cu}$ phosphor particles; (a) to (d) and non-etched milled particle (e) and magnified image of part of the latter image (f). the optical microscope in this region. It is possible that light may be generated deeper inside the phosphor particle, but it may not have found a pathway to the surface, this is contrary to the conclusions drawn by some workers that light emission is from regions near to the surface of the particle. ${ }^{24,27}$

Our previous studies of hydrochloric acid etched ACEL $\mathrm{ZnS}: \mathrm{Cu}$ phosphor particles studied using FESEM indicated that voids within the particle were clearly evident. ${ }^{11}$ Structural cavities are observed in hydrochloric acid etched samples, such as triangular shaped voids between stacks and, hexagonal basal hollows. Previously hydrochloric acid etched ACEL $\mathrm{ZnS}: \mathrm{Cu}$ phosphor particles were milled, whereby, approximately half of the bulk was removed from the samples, FESEM examples of these ACEL phosphor particles are shown in Figures 8a to $8 \mathrm{~d}$. Figures $8 \mathrm{a}$ and $8 \mathrm{~b}$ are images showing a large hexagonal shaped hydrochloric acid etched particle before and after milling respectively, viewed looking down along the [111] axis. In the image of the milled sample presented in Figure 8b numerous irregular shaped depressions with a wide size distribution are apparent. The FESEM images displayed in Figure 8c and d are an ACEL phosphor particle that has a 90 degree rotation from the previous example viewed perpendicular to the [111] plane, channels are observed to be running along the [111] axis. Some of these channels are a few micrometers across, with a significant depth and they are clearly not artifacts due to the milling process. These features may be possible pathways for some internally generated light to pass to the surface of the particle. Presented in Figure 8e and $8 \mathrm{f}$ are FESEM images of ACEL ZnS:Cu phosphor particles that have been milled without having had hydrochloric acid etching treatment a magnified image of the central particle is shown in (e). The interior of the particles have similar cavities and channels to those that had been hydrochloric acid etched. Therefore it can be stated with confidence that the etching treatment with hydrochloric acid did not produced these features, moreover similar cavities and channels are present in all synthesized ACEL $\mathrm{ZnS}: \mathrm{Cu}$ particles. Although the mechanical abrasion of the milling may have caused some removal of material, the abraded debris on and around the particles are not of a significant size to allow for the volume and depth of the voids and channels that are shown to be present within the particles in Figure 8.

During these studies on hydrochloric acid etched ACEL $\mathrm{ZnS}: \mathrm{Cu}$ phosphor particles, some were observed to undergo movement to allow them to orient with the applied AC field (when the field was originally turned on, before the adhesive had cured). It was found that when the crystal striations were not aligned parallel to some degree with the applied electric field, and these crystals were free to move, when a sufficient voltage was applied their response was instantaneous. Inter-particle collisions during the application of the electric field may also hinder and complicate the alignment of the phosphor particles. When the applied electric field was turned off, and re-applied the phosphor particles remained in their newly aligned positions. The phosphor particles also responded to a direct current electric field set up between two indium tin oxide glass electrodes. They showed initial jumping then some became stationary; others twitched and jumped then were stationary, but then jumped again as if they were trying to find a more comfortable/stable position or orientation. We could not see if the particles aligned with the field (we expect they did) as they were between two glass electrodes and moved when the top one was removed. 
In a recent paper by Medling et al. ${ }^{28}$ that describes the "local degradation of electroluminescent centers in $\mathrm{ZnS}: \mathrm{Cu}, \mathrm{Cl}$ phosphors" two figures are presented showing images of two different particles in a small ACEL devices emitting light under the influence of an applied field. The paper reported that the particles were a blue-green emitting Osram-Sylvania $\mathrm{ZnS}: \mathrm{Cu}, \mathrm{Cl}$ commercial phosphor, but did not report whether they were coated or not. The pictures were recorded using an oil immersion optical microscope. The first image (Figure 1 in the paper) shows a group of white blurred spots (of varying brightness) some with comet like tails. ${ }^{28}$ The main spots appear to be in a roughly circular formation and from a comparison of our work this particle would be aligned with its (111) orientated upwards toward the microscope aperture. As we showed earlier this alignment still allows alignment of the [111] stacking planes with the applied electric field. The particle (Figure 2 of the paper) is presented in a set of six pictures taken at one minute time intervals. This particle shows predominantly parallel lines of lights containing some bright spots, these images when compared to those presented in this paper would be of a particle that has its (111) axis perpendicular to the applied field. So in the second figure the lines of light arise between the planar stacking faults parallel to the applied field. Thus the paper shows light emission from crystals that are aligned with the applied field in each of the two possible orientations where such alignment can occur. The paper concludes that the observations only fit a degradation mechanism where damage to a $\mathrm{Cu}$ complex is rapid and can be reversed. They further conclude that a proposed degradation model in which the needles in Fischer's model might become rounded after many AC cycles cannot account for their observations. It would appear that our work although not aimed at studying the degradation per se, agrees with this papers finding.

\section{Conclusions}

We have demonstrated herein that the internal structure of the commercial and in house ACEL phosphor particles can be revealed when the particles are etched using hydrochloric acid. Such etched crystals reveal how ACEL phosphor particles orient in an applied ACEL field if free to do so. In a commercial device where the particles are fixed in a polymer matrix only the particles that are aligned or partially aligned with the applied field direction will emit light. Those that are partially orientated will emit some light, but the most emission will be observed from those crystals that are fully aligned with the applied field. These findings explain why earlier studies found none emitting crystals in their ACEL panels.

Also we have shown that the previous observations of comets (the evidence used to explain the mechanism of ACEL luminescence in powdered phosphor crystals) are in fact the result of the internal structures found within the crystals. In addition the fact that ACEL crystals can orient in an applied field shows the induced charge separation in the crystals caused by the applied field is large enough to move the crystals in the field.
Finally it is apparent from this work that the orientation of the particles to the applied field is all important to maximize the light emission. ${ }^{29}$ From our observations on normal panels up to one third of all particles are aligned completely against the field (wrong orientation) and a further third are only partially aligned and only a further third are only partially aligned with the field, hence we would expect almost twice the light output at any applied voltage if all particles were aligned. Of course suggesting how such alignment could be achieved is not straight forward.

\section{Acknowledgments}

We are grateful to the EPSRC and DTI for supporting the ACELOOP and FLCOLOUR programs, respectively. We are also grateful to the Technology Strategy Board-led Technology Program for funding the PLACES, SHAPEL, ACTIVEL and BEDS programs.

\section{References}

1. G. Blasse and B. C. Grabmaier, Luminescent Materials, Springer Verlag, Berlin (1994).

2. M. Bredol and H. S. Dieckhoff, Materials, 3, 1353 (2010)

3. G. Destriau, J. Chemie Physique, 33, 587 (1936).

4. C. Frondel and C. Palache, Science, 107, 602 (1948).

5. C. Frondel and C. Palache, Am. Miner, 35, 29 (1950).

6. D. P. Varn, G. S. Canright, and J. P. Crutchfield, Phys. Rev. B, 66, 174110 (2002).

7. D. P. Varn and G. S. Canright, Acta Cryst., A, 57, 4 (2001).

8. R. Withnall, J. Silver, T. G. Ireland, G. R. Fern, and P. J. Marsh, J. Electrochem. Soc., 156, J326 (2009).

9. M. E. Fleet, Am. Miner., 62, 540 (1977).

10. Z. Shuyuan, G. Changxin, L. Bilin, and T. Shun, Phil. Mag. A, 62, 387 (1990).

11. P. Goldberg, J. Appl. Phys., 32, 1520 (1962).

12. A. G. Fischer, J. Electrochem. Soc., 109, 1043 (1962).

13. A. G. Fischer, J. Electrochem. Soc., 110, 733 (1963).

14. J. L. Gillson and F. J. Darnell, Phys. Rev., 125, 149 (1962)

15. J. F. Waymouth and F. Bitter, Phys. Rev., 95, 941 (1954).

16. W. Lehman, J. Electrochem. Soc., 107, 657 (1960).

17. Y. Ono, N. Shiraga, H. Kadokura, and K. Yamada, Inst. Electron Inform. Commun. Eng. Tech. Rep., 89, 378 (1990)

18. C. X. Guo, S. Y. Zhang, B. L. Li, and L. Xu, J. Lumin., 40-41, 782 (1988),

19. M. Warketin, F. Bridges, S. A. Carter, and M. Anderson, Phys. Rev. B, 75, 75301 (2007).

20. S. Medling, C. France, B. Balaban, M. Kozina, Y. Jiang, F. Bridges, and S. A. Carter, J. Phys. D.: Appl. Phys., 44, 205402 (2011).

21. J. Stanley, Y. Jiang, F. Bridges, S. A. Carter, and L. Ruhlen, J. Phys.: Condens. Matter, 22, 055301 (2010).

22. G. R. Fern, T. G. Ireland, P. Harris, J. Silver, R. Withnall, A. Salimian, P. K. Santra, M. Leoni, A. Erko, A. Lennie, and C. C. Tang, Diamond Light Source Proceedings, 1, e106 (2011).

23. V. E. Oranovskij and B. A. Khmelinin, Opt. i Spectr., 7, 542 (1959).

24. A. C. Wright and I. V. F. Viney, Phil. Mag. B, 81, 279 (2001).

25. J. Silver, E. Withnall, T. G. Ireland, X. Yan, K. Saltoun, and J. J. Ojeda, Proc. $18^{\text {th }}$ International Display Workshops, (Nagoya, Japan, 6-9 December 2011) p. 731 .

26. With permission; J. A. Jaszczak, Michigan Technological University, USA.

27. N. E. Grzeskowiak and J. F. Winkel, J. Electrochem. Soc., 154, J289 (2007).

28. S. Medling, F. Bridges, and S. A. Carter, J. Lumin., 134, 251 (2013).

29. J. Silver, T. G. Ireland, P. Harris, G. R. Fern, and R. Withnall, SID Tech. Digest, 44, 224 (2013). 